\title{
Fully Integrated High-Voltage Generators with Optimized Power Efficiency
}

\author{
Doutreloigne Jan \\ Centre for Microsystems Technology (CMST), Interuniversity Microelectronics Centre (IMEC) and University of \\ Gent, Gent, Belgium \\ Email: jdoutrel@elis.ugent.be
}

Received 7 August 2014

\begin{abstract}
This paper describes how the power efficiency of fully integrated Dickson charge pumps in highvoltage IC technologies can be improved considerably by implementing charge recycling techniques, by replacing the normal PN junction diodes by pulse-driven active diodes, and by choosing an appropriate advanced smart power IC technology. A detailed analysis reveals that the combination of these 3 methods more than doubles the power efficiency compared to traditional Dickson charge pump designs.
\end{abstract}

\section{Keywords}

Charge Recycling, Dickson Charge Pump, High-Voltage Generator, Power Efficiency Optimization, Smart Power Technology

\section{Introduction}

Fully integrated charge pumps are used in a wide variety of applications, going from flash memories over dedicated display drivers to MEMS actuator drivers. Some applications ask for specific features, such as high current drive capability or very good output voltage stability. But for all of them, good overall power efficiency is of the utmost importance.

Achieving high power efficiency in fully integrated Dickson charge pumps is not straightforward due to the fact that integrated capacitors exhibit considerable parasitic capacitance to the substrate, resulting in a dramatic efficiency drop. In this paper, charge recycling techniques are presented to substantially reduce this negative effect. Replacing the standard PN junction diodes by pulse-driven DMOS transistors acting as almost ideal active diodes is another method to enhance the power efficiency. Finally, the choice of an appropriate smart power IC technology with superior transistor and integrated capacitor performance also helps to boost the efficiency. In this paper, these 3 methods are described and compared.

\section{Basic Dickson Charge Pump Design}

The basic configuration of a Dickson charge pump [1] is shown in Figure 1. It consists of a large number of identical stages, each containing a diode and a capacitor, where the bottom plates of the capacitors in consecu- 


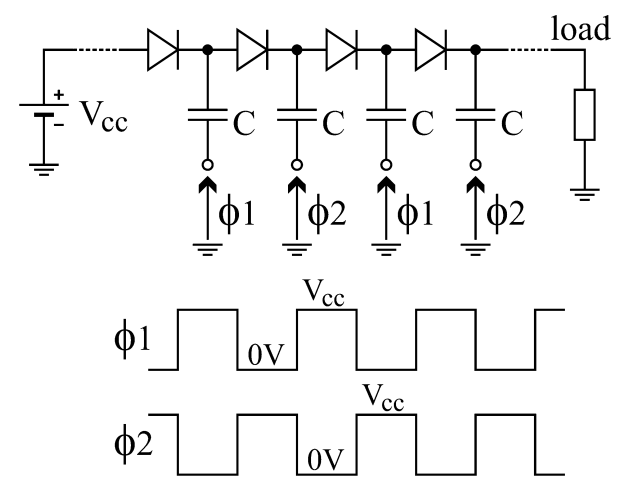

Figure 1. Basic Dickson charge pump.

tive stages are driven by 2 complementary clock signals. Charge is transferred from one capacitor to the next at the rhythm of the clock, yielding an output voltage that can be much higher than the input voltage. In a real application, a feedback control loop is added to the circuit in order to maintain the output voltage very stable and independent of system parameters or load characteristics. This can be achieved by continuously adjusting the clock frequency of the charge pump.

A major issue in integrated Dickson charge pumps is the fact that the integrated capacitors exhibit a large parasitic capacitance between their bottom plate and the normally grounded substrate of the chip, as illustrated in Figure 2.

The parasitic capacitance $C_{p}$ does not contribute to the useful operation of the circuit, but it does affect the power consumption in a negative way. Indeed, the parasitic capacitance $C_{p}$ is connected directly to the output of the clock buffers, and these buffers have to charge and discharge this parasitic capacitance constantly, resulting in additional power losses inside the transistors of the clock buffers, without having any useful impact on the circuit operation. The consequence is an extremely poor power efficiency of typically $10 \%$ to $20 \%$ in case of a voltage transformation ratio of 20, even when conventional design strategies for efficiency improvement are applied [2]-[4]. It is obvious that special measures have to be taken in order to boost the efficiency to a more acceptable level. The following 3 sections describe the 3 proposed techniques to achieve this goal.

\section{Charge Recycling}

A first approach to mitigate the power losses related to the parasitic capacitance $C_{p}$ is to employ the dedicated charge recycling technique of Figure 3 . In this configuration, the normal clock buffers are replaced by 3-state buffer circuits. Before switching the clock buffers from one state to the next (from $0 \mathrm{~V}$ to the supply voltage $V_{c c}$, or vice versa), their outputs are disabled (high-impedance output) during a very short time interval and the buffer outputs are short-circuited by means of an additional MOSFET. As a result, half of the charge that was stored on the parasitic capacitance $C_{p}$ of a particular stage will be transferred to the parasitic capacitance of the next stage. Consequently, this parasitic capacitance of the next stage does not have to be charged anymore from $0 \mathrm{~V}$ to $V_{c c}$ when its clock buffer is again enabled, but only from $V_{c c} / 2$ to $V_{c c}$. This technique yields a $50 \%$ reduction of the clock buffer power consumption that is related to the parasitic capacitance in each stage, and hence, it boosts the power efficiency effectively.

\section{Pulse-Driven Active Diodes}

A second approach focuses on the diodes that take care of the correct energy flow in each stage of the Dickson charge pump. Their threshold voltage $V_{T}$ (typically $0.5 \mathrm{~V}$ for a standard PN junction diode) causes additional power losses during the energy transfer from stage to stage, and hence, it negatively impacts the power efficiency. But there is also a combined effect of the threshold voltage $V_{T}$ of the diodes and the parasitic capacitance $C_{p}$ of the integrated capacitors. Indeed, a large value of $V_{T}$ results in a low voltage gain per stage (equal to $V_{c c}-V_{T}$ under zero-load conditions), meaning that more stages are needed to obtain a predefined output voltage. As a consequence, the total parasitic capacitance to be charged and discharged by the clock buffers increases, and the power efficiency drops. So, trying to reduce the threshold voltage $V_{T}$ of the diodes also mitigates the effect of the parasitic capacitance, and that is exactly the basis of this second technique. 


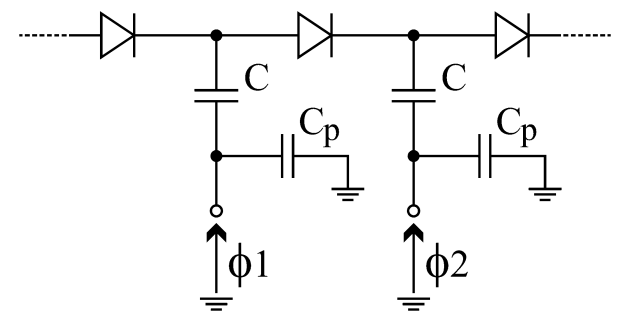

Figure 2. Presence of parasitic capacitance.
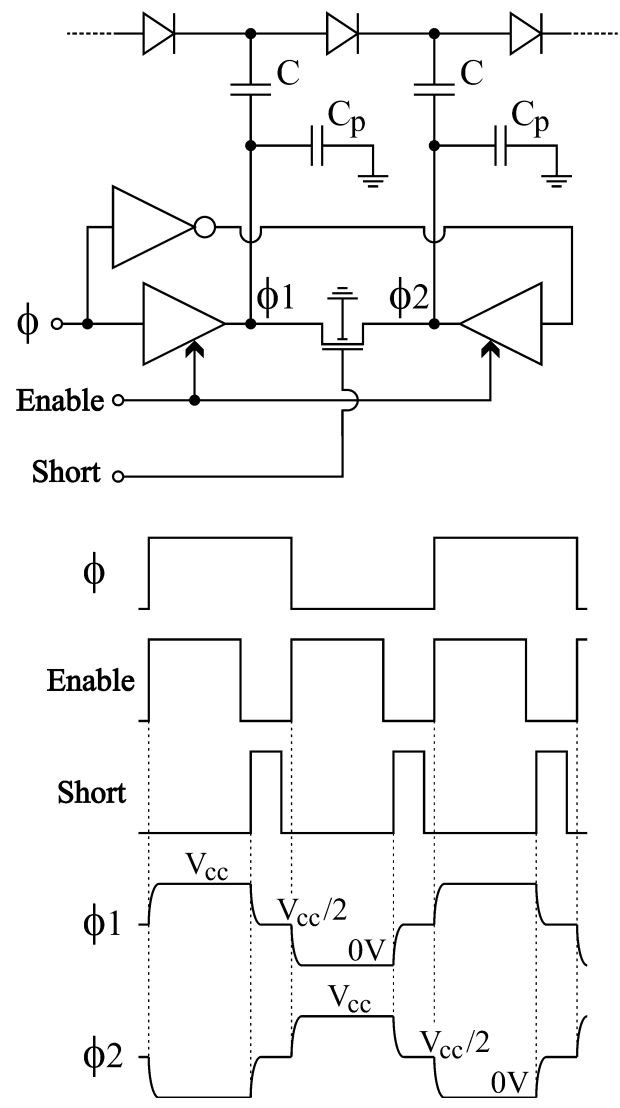

Figure 3. Charge recycling principle.

A first possibility would be to replace the standard PN junction diodes by Schottky diodes that exhibit a much lower threshold voltage. Unfortunately, high-performance Schottky diodes are not readily available in most smart power IC technologies. An interesting alternative is to replace the diodes by properly driven transistors that provide a low-resistance path during the charge transfer between capacitors of consecutive stages. In the ideal situation, the charge transfer can be completed to its full extent during the conduction time of the transistor, meaning that the equivalent $V_{T}$ value would simply be $0 \mathrm{~V}$, resulting in maximum power efficiency! A possible practical implementation of this active diode approach is shown in Figure 4.

The main charge transfer transistors are actually P-type high-voltage DMOS devices, whose built-in drainbulk diodes are oriented in exactly the same way as the diodes in the original Dickson charge pump. The operation of the circuit is fairly straightforward. When the clock signal $\phi 1$ goes high and $\phi 2$ goes low, there will be some charge transfer from the $\phi 1$-driven capacitor to the $\phi 2$-driven capacitor through the built-in drain-bulk diode of the P-type DMOS transistor between them, but this charge transfer will not be complete due to the threshold voltage of the diode. But then the pulse $\sigma 1$ is applied to the gate of an auxiliary N-type DMOS device, being the driving transistor in a level-shifter circuit, producing a voltage drop of a few volts across resistor $R$, thereby activating the P-type DMOS transistor. Hence, the low-resistance channel of this device causes the 

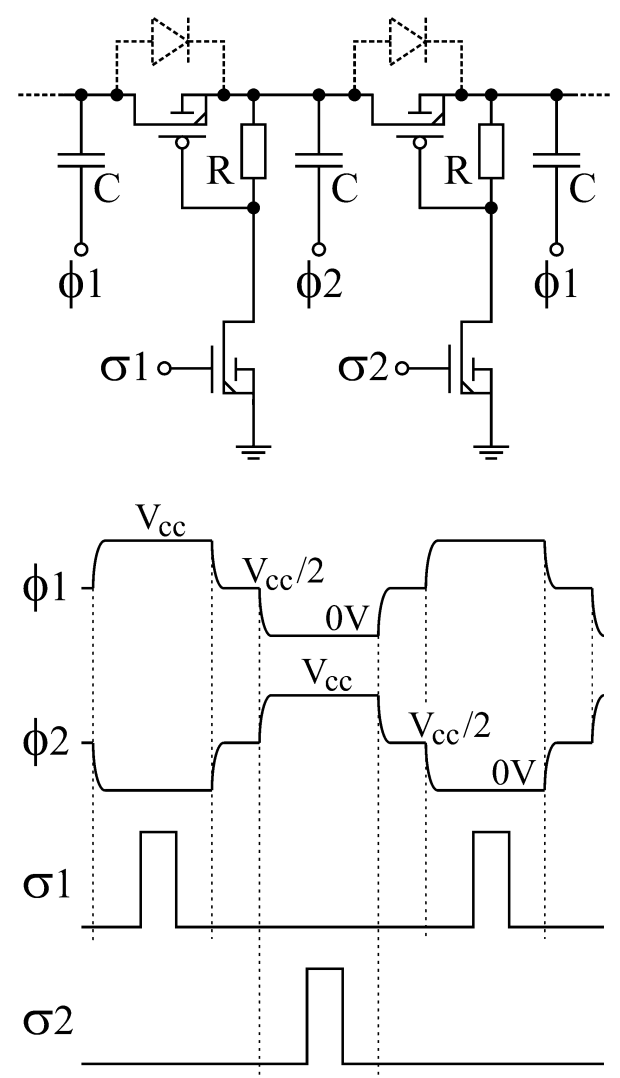

Figure 4. Pulse-driven active diodes.

charge transfer to continue until the top plates of the 2 capacitors reach exactly the same electric potential. This corresponds to the ideal situation of $V_{T}=0 \mathrm{~V}$, meaning that the P-type DMOS transistors act like almost ideal active diodes. The effect of this technique is clearly illustrated in the simulation result of Figure 5, where Vcap1 and Vcap2 are the electric potentials of the capacitor top plates in consecutive stages. The reduction of the $0.5 \mathrm{~V}$ voltage drop (the $V_{T}$ of the built-in drain-bulk diode) to $0 \mathrm{~V}$ when the P-type DMOS device is activated by the $\sigma 1$ pulse is very clear in this simulation. Also note the previously described charge recycling in the clock signals.

Although this technique looks very simple and attractive, the practical implementation and the correct component dimensioning are not straightforward at all and require a careful optimization process.

\section{Proper IC Technology Choice}

The third method to increase the power efficiency deals with a proper choice of IC technology. Of course, the specified maximum output voltage of the Dickson charge pump will set a lower limit for the high-voltage handling capability of the smart power IC technology, but also the performance of the integrated capacitors and transistors are very important selection criteria. Indeed, competitive technologies with similar voltage ratings may offer different capacitor structures with completely different values of the parasitic capacitance, resulting in significantly different power efficiency levels. Also the specific performance (on-state resistance as well as parasitic capacitance) of the P-type and N-type high-voltage DMOS transistors in the pulse-driven active diode circuit of Figure 4 will largely affect the overall power efficiency.

To illustrate the importance of the capacitor structure, we'll consider the example of the $100 \mathrm{~V} 0.7 \mu \mathrm{m} \mathrm{I}{ }^{2} \mathrm{~T}$ technology (Intelligent Interface Technology) of ON Semiconductor. Figures 6-8 show a vertical cross section of 3 different kinds of integrated capacitors in this technology. Figure 6 depicts a capacitor between a poly-silicon layer at the top and a highly doped $\mathrm{N}^{+}$implantation at the bottom, with a very thin dielectric in between. Figure 7 represents a capacitor structure between 2 poly-silicon layers, with a somewhat thicker dielectric. Finally, Figure 8 shows a sandwich structure where the shorted poly-silicon and metal 2 layers form 2 capacitors 


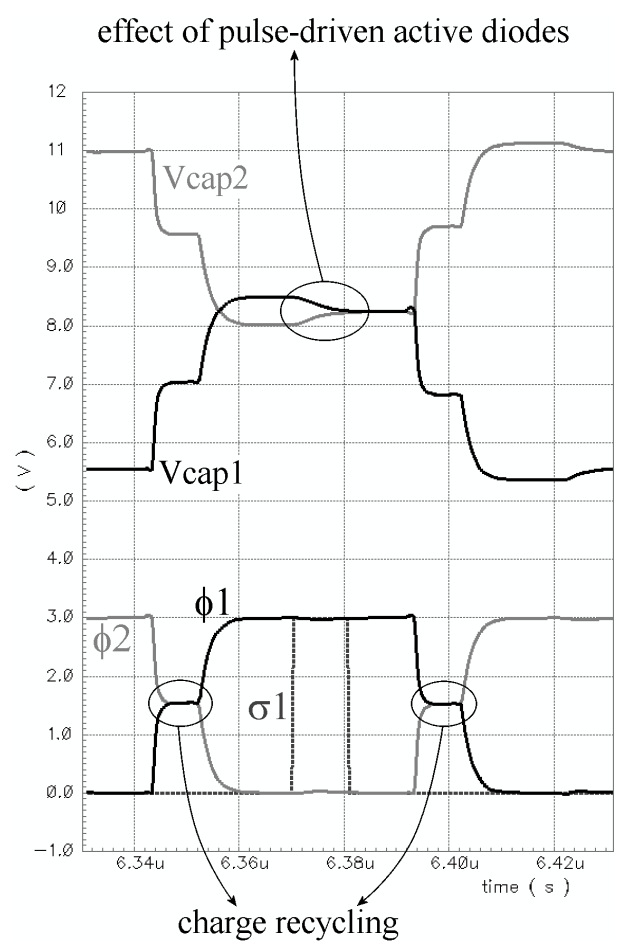

Figure 5. Simulation of the pulse-driven active diodes.

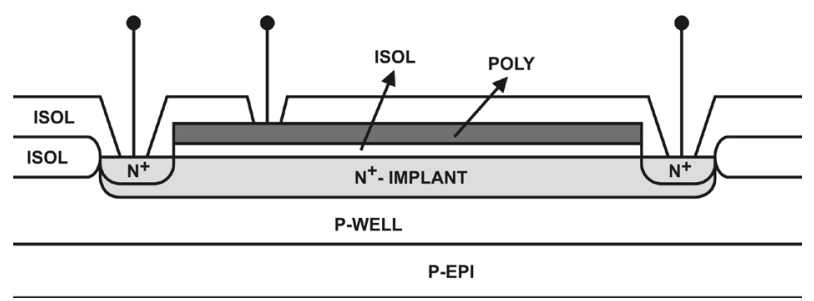

Figure 6. Poly- $\mathrm{N}^{+}$capacitor (PN capacitor).

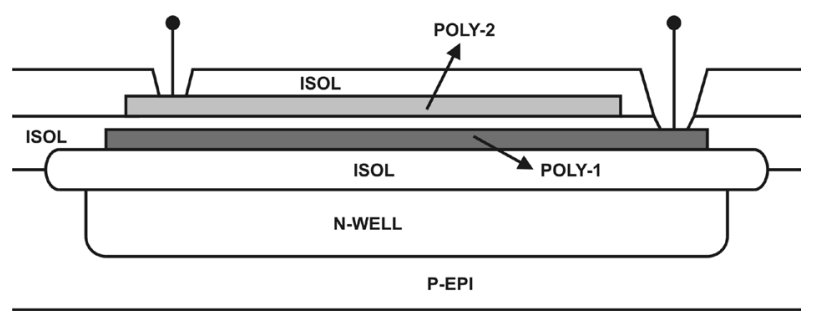

Figure 7. Poly1-poly2 capacitor (PP capacitor).

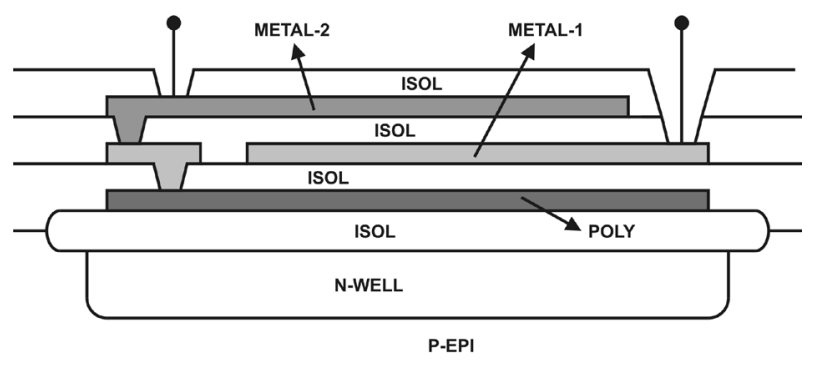

Figure 8. Metal1-metal2-Poly capacitor (MM capacitor). 
in parallel towards the metal1 layer. The dielectric in this structure is considerably thicker than in the 2 previous cases.

Although this technique looks very simple and attractive, the practical implementation and the correct component dimensioning are not straightforward at all and require a careful optimization process.

Proper IC Technology Choice

The third method to increase the power efficiency deals with a proper choice of IC technology. Of course, the specified maximum output voltage of the Dickson charge pump will set a lower limit for the high-voltage handling capability of the smart power IC technology, but also the performance of the integrated capacitors and transistors are very important selection criteria. Indeed, competitive technologies with similar voltage ratings may offer different capacitor structures with completely different values of the parasitic capacitance, resulting in significantly different power efficiency levels. Also the specific performance (on-state resistance as well as parasitic capacitance) of the P-type and N-type high-voltage DMOS transistors in the pulse-driven active diode circuit of Figure 4 will largely affect the overall power efficiency.

To illustrate the importance of the capacitor structure, we'll consider the example of the $100 \mathrm{~V} 0.7 \mu \mathrm{m} \mathrm{I}{ }^{2} \mathrm{~T}$ technology (Intelligent Interface Technology) of ON Semiconductor. Figures 6-8 show a vertical cross section of 3 different kinds of integrated capacitors in this technology. Figure 6 depicts a capacitor between a poly-silicon layer at the top and a highly doped $\mathrm{N}^{+}$implantation at the bottom, with a very thin dielectric in between. Figure 7 represents a capacitor structure between 2 poly-silicon layers, with a somewhat thicker dielectric. Finally, Figure 8 shows a sandwich structure where the shorted poly-silicon and metal2 layers form 2 capacitors in parallel towards the metal 1 layer. The dielectric in this structure is considerably thicker than in the 2 previous cases.

Based on the information from Table 1, we can easily select the most appropriate capacitor type for every individual stage in the Dickson charge pump. In the first set of stages, where the capacitor operating voltage is limited to values below $30 \mathrm{~V}$, the PP capacitor is selected as it is superior in terms of percentual parasitic capacitance. However, for the last stages with operating voltages in excess of $30 \mathrm{~V}$, the MM capacitor is the only option because of the voltage rating. The very bad corresponding percentual parasitic capacitance towards the substrate is then something we have to live with and it means that for charge pumps with an output voltage much higher than $30 \mathrm{~V}$, the overall power efficiency of the generator will be very low!

For charge pumps with very high output voltages it is therefore advisable to compare several smart power IC technologies and to select the one that offers a type of high-voltage capacitor with the lowest possible percentual parasitic capacitance towards the substrate. Take for instance the $80 \mathrm{~V} \mathrm{I}^{3} \mathrm{~T}$ technology (Improved Intelligent Interface Technology) of ON Semiconductor, which is much more advanced than the $100 \mathrm{~V} \mathrm{I}^{2} \mathrm{~T}$ technology as it is based on a $0.35 \mu \mathrm{m}$ CMOS core process instead of the older $0.7 \mu \mathrm{m}$ process. Due to the fact that this $80 \mathrm{~V} \mathrm{I} \mathrm{I}^{3} \mathrm{~T}$ technology allows much smaller metal track widths and spacings as well as 5 metal levels instead of only 2, it becomes possible to integrate multi-metal capacitors with a kind of staggered "finger"-structure design of the 2 electrodes as shown in the schematic cross-sectional view of Figure $\mathbf{9}$.

This type of capacitor design makes optimal use of the horizontal and vertical dimensions (there is capacitance between neighboring metal stripes in horizontal and vertical direction) to get the maximum capacitance for a given amount of silicon area while keeping the parasitic capacitance towards the substrate to a minimum. This is clearly evidenced in Table 2, comparing the performance of the metal1-metal2-poly capacitor (MM) in the $100 \mathrm{~V} 0.7 \mu \mathrm{m} \mathrm{I}^{2} \mathrm{~T}$ technology and the multi-metal "finger" capacitor (MF) in the $80 \mathrm{~V} 0.35 \mu \mathrm{m} \mathrm{I}^{3} \mathrm{~T}$ technology. As could be expected, the high-voltage MF capacitors in the $80 \mathrm{~V} 0.35 \mu \mathrm{m} \mathrm{I}^{3} \mathrm{~T}$ technology exhibit a 4 times lower percentual parasitic capacitance than the high-voltage MM capacitors in the $100 \mathrm{~V} 0.7 \mu \mathrm{m} \mathrm{I}{ }^{2} \mathrm{~T}$ technology. This makes the $80 \mathrm{~V} 0.35 \mu \mathrm{m} \mathrm{I} \mathrm{I}^{3} \mathrm{~T}$ technology the preferred choice for integrating high-voltage Dickson charge pumps with maximum power efficiency.

Table 1. Comparison of different capacitor types in the $100 \mathrm{~V} 0.7 \mu \mathrm{m} \mathrm{I} \mathrm{I}^{2} \mathrm{~T}$ technology.

\begin{tabular}{cccc}
\hline Capacitor type & Max. voltage $(\mathrm{V})$ & Specific capacitance $\left(\mathrm{fF} / \mu^{2}\right)$ & Parasitic capacitance $\left(\mathrm{fF} / \mu \mathrm{m}^{2}\right)$ \\
\hline PN & 12 & 0.75 & $0.27(36 \%)$ \\
PP & 30 & 0.345 & $0.079(23 \%)$ \\
MM & 100 & 0.091 & $0.057(63 \%)$ \\
\hline
\end{tabular}




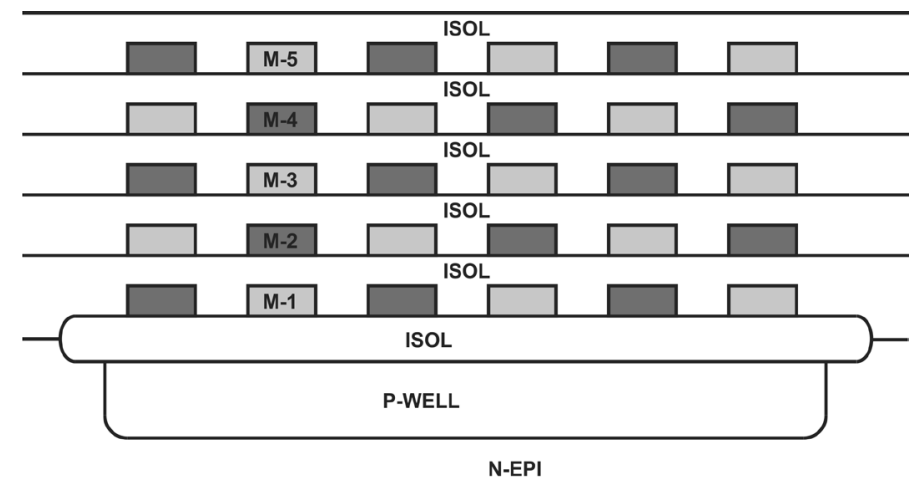

Figure 9. Multi-metal capacitor with staggered “finger”-structure design (MF capacitor).

Table 2. Comparison of capacitor performance between the $100 \mathrm{~V} 0.7 \mu \mathrm{m} \mathrm{I}{ }^{2} \mathrm{~T}$ and $80 \mathrm{~V} 0.35$ $\mu \mathrm{m} \mathrm{I}{ }^{3} \mathrm{~T}$ technologies.

\begin{tabular}{cccc}
\hline Capacitor + technology type & $\begin{array}{c}\text { Max. voltage } \\
(\mathrm{V})\end{array}$ & $\begin{array}{c}\text { Specific capacitance } \\
\left(\mathrm{fF} / \mu^{2}\right)\end{array}$ & $\begin{array}{c}\text { Parasitic capacitance } \\
\left(\mathrm{fF} / \mu \mathrm{m}^{2}\right)\end{array}$ \\
\hline MM 100 V 0.7 $\mu \mathrm{m} \mathrm{I}^{2} \mathrm{~T}$ & 100 & 0.091 & $0.057(63 \%)$ \\
MF 80 V 0.35 $\mu \mathrm{m} \mathrm{I}^{3} \mathrm{~T}$ & 80 & 0.32 & $0.047(15 \%)$ \\
\hline
\end{tabular}

When comparing smart power IC technologies, also differences in high-voltage transistor structures and transistor characteristics may be observed. As an example, Table 3 compares the same $100 \mathrm{~V} 0.7 \mu \mathrm{m} \mathrm{I}{ }^{2} \mathrm{~T}$ and $80 \mathrm{~V}$ $0.35 \mu \mathrm{m} \mathrm{I} \mathrm{I}^{3} \mathrm{~T}$ technologies in terms of specific on-state resistance of the main high-voltage P-type and N-type DMOS transistors. It is clear from this table that the high-voltage DMOS devices in the $80 \mathrm{~V} 0.35 \mu \mathrm{m} \mathrm{I}{ }^{3} \mathrm{~T}$ technology have a roughly 3 times lower on-state resistance, which means that the duration of the control pulses $\sigma 1$ and $\sigma^{2}$ for activating the level-shifters in the pulse-driven active diode circuit of Figure $\mathbf{4}$ can be chosen much shorter than in the case of the $100 \mathrm{~V} 0.7 \mu \mathrm{m} \mathrm{I}^{2} \mathrm{~T}$ technology, resulting in less power dissipation inside the levelshifters, and hence, improved overall power efficiency.

From the discussions of Table 2 and Table 3, we conclude that a careful comparison and selection of smart power IC technologies is of the utmost importance to achieve the maximum power efficiency in integrated high-voltage Dickson charge pumps!

\section{Impact of the 3 Proposed Techniques}

To illustrate the effect of the 3 techniques we proposed for boosting the power efficiency of integrated highvoltage Dickson charge pumps, a comparison is made for 1 specific application: a monolithic driver chip for a $60 \mathrm{~V}$ bistable nematic Liquid Crystal Display (LCD). For that purpose an integrated Dickson charge pump is needed, capable of transforming a $3 \mathrm{~V}$ supply voltage into a $60 \mathrm{~V}$ output voltage with a maximum output current rating of $200 \mu \mathrm{A}$. In every design (i.e. for each implementation of the proposed techniques), a clock frequency of $10 \mathrm{MHz}$ and a capacitor of $12 \mathrm{pF}$ in each stage of the charge pump were assumed, and the number of stages was chosen in such a way that the specified $60 \mathrm{~V}$ output voltage was within reach for the maximum output current value of $200 \mu \mathrm{A}$. The results of this comparative study can be observed in Figure 10, showing the power efficiency as a function of the output current for several design cases.

From this graph, the importance of the proposed efficiency boosting techniques becomes very clear. Combining the charge recycling feature, the pulse-driven active diode technique and the use of an advanced smart power technology with enhanced high-voltage capacitor and transistor performance more than doubles the power efficiency compared to the traditional Dickson charge pump configuration (no charge recycling, standard PN junction diodes and the use of an older IC technology with less performing high-voltage capacitors and transistors), and this over the whole useful output current range. It is in fact very remarkable that a power efficiency of almost $50 \%$ can be reached at the optimal operating point for this very high voltage transformation ratio from $3 \mathrm{~V}$ to $60 \mathrm{~V}$ in a fully integrated high-voltage generator! 


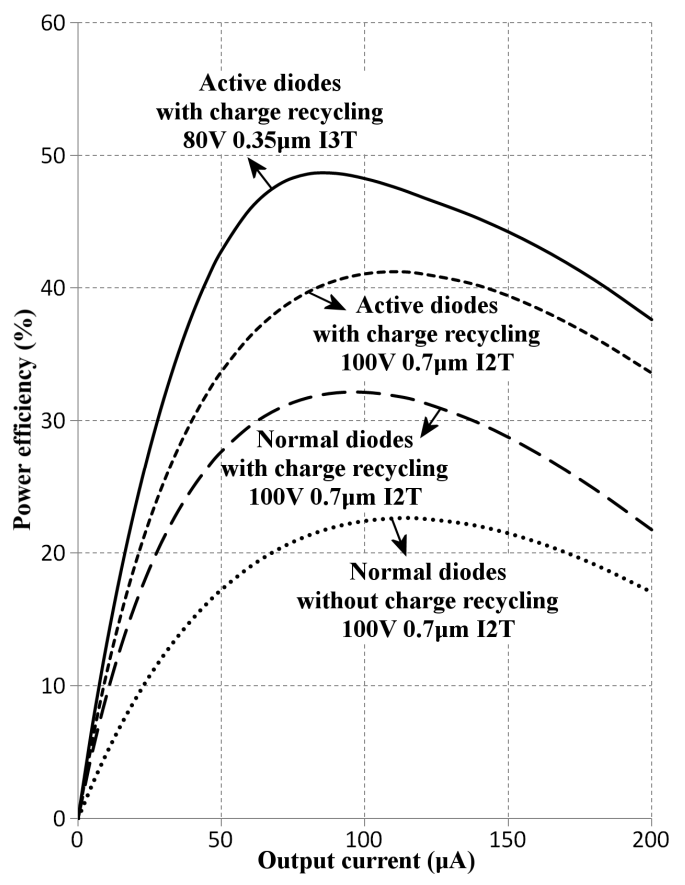

Figure 10. Impact of the 3 proposed techniques on the power efficiency versus output current characteristic.

Table 3. Comparison of high-voltage transistor performance between the $100 \mathrm{~V} 0.7 \mu \mathrm{m} \mathrm{I}{ }^{2} \mathrm{~T}$ and $80 \mathrm{~V} 0.35 \mu \mathrm{m} \mathrm{I}{ }^{3} \mathrm{~T}$ technologies.

\begin{tabular}{|c|c|c|c|}
\hline Technology & Transistor type & Max. voltage (V) & Specific on-state resistance $\left(\Omega \mathrm{mm}^{2}\right)$ \\
\hline \multirow{2}{*}{$100 \mathrm{~V} 0.7 \mu \mathrm{m} \mathrm{I}{ }^{2} \mathrm{~T}$} & N-type DMOS & 100 & 0.76 \\
\hline & P-type DMOS & 90 & 1.32 \\
\hline \multirow{2}{*}{$80 \mathrm{~V} 0.35 \mu \mathrm{m} \mathrm{I} \mathrm{I}^{3} \mathrm{~T}$} & N-type DMOS & 80 & 0.26 \\
\hline & P-type DMOS & 80 & 0.47 \\
\hline
\end{tabular}

\section{Conclusion}

Three methods for increasing the power efficiency of fully integrated Dickson charge pumps were presented and analyzed: the use of charge recycling techniques, the replacement of standard PN junction diodes by pulse-driven active diodes, and the use of an advanced smart power IC technology with high-performance integrated high-voltage capacitors and transistors. Data from specific design cases provide a clear proof of the positive impact of these 3 methods. It is shown that the power efficiency is more than doubled compared to traditional Dickson charge pump configurations.

\section{References}

[1] Dickson, J.F. (1976) On-Chip High-Voltage Generation in MNOS Integrated Circuits Using an Improved Voltage Multiplier Technique. IEEE Journal of Solid-State Circuits, 11, 374-378. http://dx.doi.org/10.1109/JSSC.1976.1050739

[2] Di Cataldo, G. and Palumbo, G. (1996) Design of an N-th Order Dickson Voltage Multiplier. IEEE Transactions on Circuits and Systems-I: Fundamental Theory and Applications, 43, 414-417. http://dx.doi.org/10.1109/81.502213

[3] Palumbo, G., Pappalardo, D. and Giabotti, M. (2002) Charge-Pump Circuits: Power-Consumption Optimization. IEEE Transactions on Circuits and Systems I: Fundamental Theory and Applications, 49, 1535-1542.

[4] Palumbo, G., Pappalardo, D. and Giabotti, M. (2001) Modeling and Minimization of Power Consumption in Charge Pump Circuits. Proceedings of the 2001 IEEE International Symposium on Circuits and Systems (ISCAS), 402-405. http://dx.doi.org/10.1109/TCSI.2002.804544 